\title{
Diffuse Symmetric Cerebral Calcifications: An Emerging Clinical Pivot
}

\author{
Jacob R. Hascalovici, Constantine Farmakidis
}

Keywords: Albright hereditary osteodystrophy, Cerebral calcification, Primary familial brain calcification, Pseudohypoparathyroidism

A 53-year-old man with a history of depression and polysubstance abuse presented to the emergency department for neurologic evaluation after a fall and mild head trauma. Computed tomography scan of the head showed prominent symmetric basal ganglia, thalamic, cerebellar hemisphere, occipital cortex, and subcortical U fiber hyperdensities (200-400 Hounsfield units) consistent with calcium deposition (Figure 1). Serum studies were notable for hypocalcemia, elevated parathyroid hormone (PTH) levels twice the upper limit of normal, normal phosphorus and magnesium, and vitamin D deficiency. Hand X-rays showed shortening of the bilateral fourth and fifth metacarpals (brachydactyly), indicating a likely diagnosis of Albright hereditary osteodystrophy and pseudohypoparathyroidism, type I. Beyond his previous psychiatric history, the neurologic history and examination were unremarkable.

\section{DisCUSSION}

We present a case of bilateral striopallidodentate, occipital, cerebellar, and U fiber calcifications, PTH resistance, and osteodystrophic changes most similar with pseudohypoparathyroidism, type I, and Albright hereditary osteodystrophy. The concurrent vitamin D deficiency and well-established heterogeneity of
PTH resistance disorders likely explain the normal serum phosphorous levels. ${ }^{1}$

More pronounced seemingly idiopathic symmetric cerebral calcifications are classified as either familial or sporadic. These are distinct from the incidental, nonspecific, and age-dependent basal ganglia calcifications seen on imaging with an estimated frequency of $1 \%$ to $20 \% .^{2}$ The familial form of symmetric cerebral calcification is now known as primary familial brain calcification, for which causative mutations have been identified in four genes to date. ${ }^{2,3}$ Primary familial brain calcification is most frequently autosomal dominant, and neurologic manifestations are notably variable and include movement disorders, cognitive impairment, cerebellar dysfunction, seizures, and psychiatric symptomatology. ${ }^{2}$ Other major causes of diffuse symmetric cerebral calcifications are disorders of PTH metabolism as in this case, along with infectious causes and radiation exposure (Table 1).

This case highlights how improved understanding of the human genetics and diverse clinical features in patients with diffuse symmetric cerebral calcifications can clarify the differential diagnosis and clinical approach to this radiologic finding. Evaluation of patients with diffuse cerebral calcifications should include a careful family history; a laboratory evaluation including

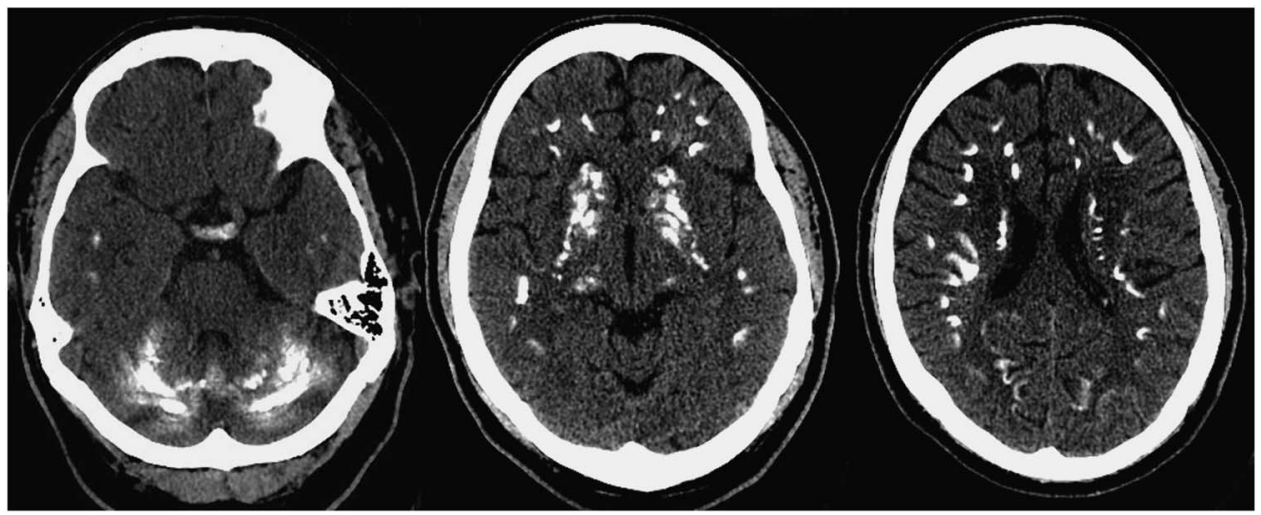

Figure 1: Pronounced bilateral striopallidodentate, occipital, cerebellar, and U fiber calcifications.

\footnotetext{
From the Saul R. Korey Department of Neurology, Montefiore Medical Center, The Albert Einstein College of Medicine, Bronx, New York. Received August 7, 2015. Final Revisions Submitted May 24, 2016. Date of Acceptance August 8, 2016.

Correspondence to: Jacob R. Hascalovici, Department of Neurology, Montefiore Medical Center, The Albert Einstein College of Medicine, 3351 Steuben Ave., 3rd Floor, Bronx, NY, 10467. Email: jhascalo@montefiore.org
} 
Table 1: Differential diagnosis for diffuse symmetric cerebral calcifications

\begin{tabular}{l|l}
\hline \multirow{2}{*}{ Endocrine } & Hypoparathyroidism \\
\cline { 2 - 2 } & Pseudohypoparathyroidism \\
\hline \multirow{2}{*}{ Infection } & Neurocysticercosis \\
\cline { 2 - 2 } & TORCH infection \\
\cline { 2 - 2 } & HIV \\
\hline Primary familial brain calcification & SLC20A2, PDGFRB, PDGFB, XPR1 \\
\hline Radiation & Mineralizing microangiopathy \\
\hline
\end{tabular}

TORCH = toxoplasmosis, other (syphilis, varicella zoster, parvovirus

B19), rubella, cytomegalovirus, and herpes.

serum calcium, phosphate and urinary calcium, PTH, thyroidstimulating hormone, and endocrine consultation as needed; neurologic screening for cognitive, behavioral, and extrapyramidal illness; and, if necessary, referral to movement disorder specialists in a quaternary care center.

\section{Disclosures}

None.

\section{REFERENCES}

1. Mantovani G. Clinical review: pseudohypoparathyroidism: diagnosis and treatment. J Clin Endocrinol Metab. 2011;96: 3020-30.

2. Tadic V, Westenberger A, Domingo A, Alvarez-Fischer D, Klein C, Kasten M. Primary familial brain calcification with known gene mutations: a systematic review and challenges of phenotypic characterization. JAMA Neurol. 2015;72: 460-7.

3. Legati A, Giovannini D, Nicolas G, et al. Mutations in XPR1 cause primary familial brain calcification associated with altered phosphate export. Nat Genet. 2015;47:579-81. 